\title{
A Preliminary Investigation of Training Order for Introducing NextGen Tools
}

\author{
R. Conrad Rorie ${ }^{1}$, Ariana Kiken ${ }^{1}$, Corey Morgan ${ }^{1}$, Sabrina Billinghurst ${ }^{1}$, \\ Gregory Morales ${ }^{1}$, Kevin Monk ${ }^{1}$, Kim-Phuong L. Vu ${ }^{1}$, Thomas Strybel ${ }^{1}$, \\ and Vernol Battiste ${ }^{2}$ \\ ${ }^{1}$ California State University Long Beach \\ Center for Human Factors in Advanced Aeronautics Technologies \\ 1250 N Bellflower Blvd. Long Beach, CA 90840, USA \\ ${ }^{2}$ San Jose State University Foundation and NASA Ames Research Center \\ Moffett Field, CA 94035, USA \\ robert_rorie@yahoo.com, \\ \{aegkiken, coreyandrewmorgan, sabrinabillinghurst, \\ gregory.morales\} @gmail.com, kjmonk8@yahoo.com, \\ $\{$ kvu8, tstrybel\}@csulb.edu, vernol.battiste-1@nasa.gov
}

\begin{abstract}
Eleven students enrolled in a 16-week radar simulation course were trained on current-day and NextGen tools. The order of the training was manipulated so that half of the students received current-day training first, followed by the training on NextGen tools, while the remaining students received training on the NextGen tools first, followed by current-day training. This paper reports data from the debriefing sessions following the conclusion of the course, with the intent of determining students' reaction to the training order and their comments and suggestions for future training schedules. Results indicated that future training should start with current-day procedures and delay the introduction of NextGen tools until trainees have established fundamental air traffic management skills.
\end{abstract}

Keywords: ATC, training, part-task, NextGen.

\section{Introduction}

The Next Generation Airspace Transportation System (NextGen) will replace the current air traffic management (ATM) system in the U.S. NextGen is being developed to allow the national air space to handle 2-3X current-day traffic levels in response to estimated increases in air travel in the US and world-wide by 2025 [1]. One limitation of the current ATM system is the amount of aircraft that can be safely handled by air traffic controllers (ATC) with the existing tools. NextGen intends to automate specific air traffic roles and responsibilities in order to reduce controller workload and increase their capacity to handle more aircraft. Tasks that are under consideration for automation in NextGen include conflict detection and conflict resolution [2]. In addition, tools and technologies are being considered to provide controllers with the capability of making route modifications that can be uplinked directly to the flight deck [3]. 
The introduction of NextGen concepts of operations, tools, and technologies need to be evaluated in terms of their effectiveness. As such, many studies and simulations have been conducted to test potential concepts and technologies for NextGen [4, 5, 6]. One area that has not received much attention, though, is that of training NextGen tools and procedures. Currently, ATC training is typically based on Air Traffic Basics courses provided by Air Traffic Collegiate Training Initiative programs followed by intensive training at the FAA Academy in Oklahoma City [7, 8]. After completing the basic training, controllers are then sent to a location (e.g., Tower, TRACON, Center) to receive on-site training through an apprenticeship model [7]. The apprenticeship can last from months to years. There has been some evidence that simulation training can reduce on the job training time [9]. In the present study, we examine whether the order in which NextGen tools are introduced into training can affect the learning of students who are pursuing careers in air traffic control.

Although the effectiveness of NextGen technologies needs to be tested with experienced controllers for operational validity, students are a necessary source of research participants because they will eventually become NextGen operators. $\mathrm{Vu}$ et al. [10] showed that highly-trained students in a specific sector can show analogous performance to retired controllers who were only given a 1-day training session to familiarize themselves with a new sector. Moreover, students showed more willingness to try new technologies than experienced controllers. One reason for higher acceptance of new technologies among students is that the students have grown up in an era where technology is dominant in everyday activities. Future ATC training will need to incorporate the training of NextGen concepts and technologies to fully prepare the workforce for the upcoming transformation. This study is an initial step toward that goal by examining how the order of introduction to NextGen tools affects student ATM learning. Results from this preliminary study can inform future research on how to best introduce NextGen technologies into the current-day training paradigm.

Schneider [11] described air traffic controlling as a "high performance skill", defined as a task requiring more than 100 hours of training, producing high failure rates, and exhibiting qualitative differences between the performances of novices and experts. As such, training programs need to be designed to be as effective as possible in order to promote successful completion of their requirements. Sohn et al. [12] noted that, when learning complex skills, it can be helpful to break down high-level tasks into their component parts, otherwise known as part-task training. They reasoned that becoming fluent on the component tasks increases the chances of becoming fluent on the overall task. There have been many demonstrations of parttask training compared to whole-task training in different domains [13, 14, 15]. Young et al. [16] showed that the difficulty participants experienced in training can also influence their performance later. In their study, an unrelated secondary task was added to increase the task's difficulty during training. Increased difficulty during training was found to lead to better retention of skills over time. With regard to NextGen training, these results suggest that more difficult skills should be learned first, followed by easier skills. To test this hypothesis, we vary the order of two air traffic management components, one involving the learning of current ATM skills and the other involving the learning with NextGen tools that automate the task of conflict detection and provide tools in support of conflict resolution. 
Current-day air traffic management skills include detecting aircraft in conflict by projecting aircraft trajectory and speed, using strategies for the separation of aircraft, techniques for ensuring safe merging and spacing of aircraft, and using strategies for overall sector management through the exclusive use of voice communications. Introduction of NextGen technologies into ATC training will necessitate instruction on several new, transformative tools such as Data Comm, conflict detection, and conflict probes. Data Comm, a text-based communication system between controllers and pilots, will significantly reduce the amount of voice communications made between air traffic control and pilots. Although the reduction of voice communication should reduce operator workload, the use of Data Comm will require the training of controllers on Data Comm commands. Conflict detection tools will automatically alert controllers of potential conflicts for pairs of aircraft that are equipped with NextGen technologies. Enabling conflict detection will substantially reduce controller workload since they will not need to scan for conflicts between the equipped aircraft. Conflict probes will assist controllers in the task of conflict resolution by providing them cues regarding whether a flight plan change is conflict-free or not. Again, conflict probes should reduce controller workload and cognitive demands. However, studies have shown that tools that decrease operator workload may take operators "out of the loop" and reduce their situation awareness [17]. Low levels of situation awareness can then lead to errors, especially when automation fails and the operator must perform all tasks manually [18].

The present paper is based on a larger study examining whether the order in which student controllers are trained with current day and NextGen procedures affects their performance, situation awareness and workload. This paper reports data from debriefing sessions of the larger study, with the intent of determining students' reaction to the training order, their assessment about how the training order affected their overall learning, and their comments and suggestions for future training schedules. A content analysis was performed on transcripts of the debriefing sessions and written notes by an experimenter. Due to the small sample size and preliminary nature of this training study, no formal analyses were run. Instead, we provide a qualitative summary of the participants' responses.

\section{Method}

\subsection{Participants}

Eleven students enrolled in a 16-week, radar simulation course through California State University Long Beach's Center for Human Factors in Advanced Aeronautics Technologies (CHAAT) participated. All students were enrolled in an aviation program at Mount San Antonio College (Mt SAC), and are pursuing careers in air traffic control. Students had an average of 1.5 years of study in their program and have all completed a course in the Air Traffic Control Environment at Mt SAC, which includes topics of aircraft characteristics, air traffic procedures, and phraseology.

\subsection{Training}

All students received a minimum of 6 hours per week of training on managing traffic of enroute sectors ZID 91 and 81. Traffic in the sector consisted of arrivals and 
departures to/from Louisville Standiford International Airport, as well as overflights. Two of the 6 training hours were dedicated to general air traffic management skills and airspace (en route) operations in a classroom setting taught by a retired, radarcertified controller. The other 4 hours were dedicated to hands-on radar simulation training in the CHAAT simulation lab using the MultiAircraft Control System (MACS) software developed by the Airspace Operations Laboratory at NASA Ames Research Center [19].

All students received instruction on the MACS software and on procedures and strategies for managing traffic in the sector. The students were divided into two separate training groups, differing on the timing of exposure to advanced ATC tools. For the current-day procedures, students were taught traffic management techniques for conflict detection and strategies for judging the threat level of potential conflicts. In addition, they were instructed on point-to-point vectoring techniques for safety and efficiency, and the proper phraseology for communicating with aircraft in their sector. For the NextGen tools procedures, students were trained on how to use Data Comm commands for issuing clearances as well as how to use the conflict alerting and conflict probes for Data Comm-equipped aircraft to detect and resolve conflicts. All students were also taught the procedure for taking handoffs and for handing off aircraft using voice or Data Comm, depending on the training condition.

The Current Day-First training group received training on air traffic management using only voice communications during Weeks 2-7 of the laboratory component of the course and received training with NextGen tools (Data Comm and the advanced conflict detection and resolution tools) during Weeks 9-15. Students in the NextGenFirst training group received hands-on training with Data Comm and the advanced ATC tools during Weeks 2-7 and received current-day instruction without the advanced tools during Weeks 9-15. Both groups received mixed training the week before two sets of experimental trials, which occurred at the end of Weeks 8 and 16 . The experimental trials utilized a mixed equipage environment with Data Commequipped and voice-only aircraft. At the end of the 16-week second test session, participants were debriefed on the purpose of the simulation and were asked to provide feedback regarding the training design. The conversations were recorded, with permission of the participants, and then were subsequently transcribed for the present analysis.

\subsection{Apparatus}

Participants performed their experimental trials within the same simulation environment taught during the hands-on portion of their training. MACS is a medium fidelity computer application simulating a radar screen of ZID Sector 91. It accommodates both air traffic control and pilot operations. All aircraft were piloted by "pseudopilots" trained specifically on MACS to provide a realistic traffic environment for controllers. Communication between controllers and pilots was provided by a voice server station, allowing communication via push-to-talk headsets [20]. 


\section{Results and Discussion}

Each of the eleven subjects was asked at the end of the 16-week simulation course to comment on the effectiveness of the training order in developing their ATM skills. There were six subjects in the Current Day-First condition (i.e., voice first) and five subjects in the NextGen-First condition. All six subjects in the Current Day-First condition claimed that receiving verbal training before Data Comm training was highly beneficial in developing their ATM skills and controller-pilot communication skills. Perhaps more telling was that all five participants in the NextGen-First condition reported that their order of training was ineffective, stating that it would have been more beneficial to receive the training on manual conflict detection and voice communication first. The particular themes that developed during the debriefing sessions are discussed in more detail below.

\subsection{Perceived Effectiveness of Training Order}

All students indicated that the most important skills they needed for ATM, including how to scan the scope for conflicts, predict flight trajectories, make speed projections, and make effective use of strategies taught in the classroom for modifying routes, were also the most difficult to learn. Therefore, they all reported that current day procedures should be taught first and be given the most emphasis (about threequarters instead of half of the class) during training. This finding is consistent with the difficulty of training hypothesis Young et al. [16] in that the more difficult tasks should be trained first because it leads to better transfer of skill and retention of learning.

Training the students with current-day procedures first allows them to acquire fundamental ATM skills. In particular, the task of manually scanning the scope for conflicting aircraft was the primary area that the students reported needing extended training. Since this critical task was only practiced during the Current Day-First condition, those in the NextGen-First condition reported feeling unprepared to manage traffic for their first test halfway through the course. Participants also claimed that training on the NextGen tools required less attention and therefore caused trainees to become passive as they let the computer do the majority of the work for them. Several students in that group reported that the passivity led them to "slack off" and not critically monitor their environment. However, students in the Current DayFirst condition said they continued to monitor traffic and tried to detect conflicts before the conflict alerting system notified them. As such, they were not as reliant on the conflict detection technology as the NextGen-First condition. More generally, all students felt that practice with current day tasks was the most effective at developing a foundation for ATM skills as a whole.

Participants in the NextGen-First condition claimed they felt unprepared in the mid-term testing for even routine tasks such as acknowledging aircraft check-ins and giving frequency changes. The added workload needed for them to perform these routine tasks decreased their ability to perform the more critical tasks of verbally issuing commands to aircraft and monitoring the scope for conflicting traffic. These 
students also reported that the advanced tools training emphasized the computer system and interface rather than the actual traffic separation and monitoring techniques. Due to this fact, when students in the NextGen-First condition made the switch to the current-day training in the second half of the study, they felt as if they were starting the class all over again. In other words, there was little transfer of training. This echoes the sentiment of the Current Day-First condition, which stated that current-day procedures provide a more supportive foundation for learning ATC tasks. One student noted feeling held back by beginning his training with NextGen tools. He remarked that training with NextGen tools contradicted the classroom training because those lessons taught strategies for detecting conflicts manually; however, during hands-on training with the advanced tools, students were told to refrain from manual conflict detection because the computer would perform that task for them. In this sense, the student was not able to put into practice what they learned in the classroom component of the course.

Most students, irrespective of training condition, indicated that the advanced tools were easy for them to learn and required little focused attention to use. It is not surprising, then, that participants felt eight weeks of training on NextGen tools became redundant. It is important to note, however, that the students did agree that NextGen tools were worthy of some extended training. In their opinion, the inevitable introduction of NextGen tools into the ATC domain necessitates sufficient levels of training in order to bring them up to a proficient level of performance. They also noted that NextGen tools did provide them with novel ways to separate traffic and offload some of their workload by reducing their responsibility for predicting potential conflicts.

\subsection{Limitations and Suggestions for Future Training}

This study was a preliminary investigation of how the order in which NextGen tools are introduced affects student learning of ATM tasks. Based on feedback from students in the course, it is recommended that current-day ATM techniques be trained prior to the introduction of NextGen tools. However, this recommendation assumes that NextGen air traffic will consist of both equipped and unequipped aircraft, a feature of near term NextGen. As more aircraft become equipped with NextGen tools there may be less need for learning current-day ATM techniques.

The results from this study were only intended to provide an initial input to research on training of NextGen tools. In fact, we only tested the introduction of three specific NextGen tools, so the findings may not generalize to other NextGen technologies. Also, it should be noted that the training of current-day and NextGen tools occurred only in an environment with all voice or all NextGen equipped aircraft, respectively. It may be the case that introducing NextGen tools early, in a mixed equipage environment, may increase the students' ability to acquire fundamental ATM skills while benefiting from the NextGen capabilities. In addition, other training schedules should be examined in future investigations. 
Acknowledgements. This study was supported by NASA cooperative agreement NNX09AU66A, Group 5 University Research Center: Center for the Human factors in Advanced Aeronautics Technologies (Brenda Collins, Technical Monitor).

\section{References}

1. Joint Planning and Development Office. Concept of Operations for the Next Generation Air Transportation System Version 1.2 (2007),

http: / / www . jpdo.gov/library/NextGenConopsv12.pdf

2. Prevot, T., Homola, J., Mercer, J.: Human-in-the-Loop Evaluation of Ground-Based Automated Separation Assurance for NextGen. In: Congress of International Council of the Aeronautical Sciences Anchorage, Anchorage, AK (2008)

3. Strybel, T.Z., Vu, K.-P. L., Bacon, L.P., Kraut, J., Battiste, V., Johnson, W.W.: Diagnosticity of an Online Query Technique for Measuring Pilot Situation Awareness in NextGen. In: Digital Avionics Systems Conference, pp. 4.B.1-1-4.B.1-12 (2010)

4. Ligda, S.V., Johnson, N., Lachter, J., Johnson, W.W.: Pilot Confidence with ATC Automation Using Cockpit Situation Display Tools in a Distributed Traffic Management Environment. In: Salvendy, G., Smith, M.J. (eds.) HCI International 2009. LNCS, vol. 5618, pp. 816-825. Springer, Heidelberg (2009)

5. Dao, A.V., Lachter, J., Battiste, V., Brandt, S.L., Vu, K.-P.L., Strybel, T.Z., Ho, N., Martin, P., Johnson, W.W.: Automated Spacing Support tools for Interval Management Operations during Continuous Descent Approaches. In: Human Factors and Ergonomics Society Annual Meeting Proceedings, pp. 21-25 (2010)

6. Dwyer, J.P., Landry, S.: Separation Assurance and Collision Avoidance Concepts for the Next Generation Air Transportation System. In: Salvendy, G., Smith, M.J. (eds.) Proceedings of the Symposium on Human Interface. LNCS, pp. 748-757. Springer, Heidelberg (2009)

7. Morrison, J.E., Fotohui, C.H., Broach, D.: A Formative Evaluation of the Collegiate Training Initiative. Technical paper DOT/FAA/Am-96/6, FAA Civil Aeromedical Institute, Oklahoma City (1996)

8. Cavcar, A., Cavcar, M.: New Directions for ATC Training. Int. J. Aviat. Psychol. 14, 135150 (2004)

9. Yacef, K., Alem, L.: Intelligent Tutoring Systems: Student and Expert Modelling for Simulation-based Training. In: Frasson, C., Gauthier, G., Alan, L. (eds.) ITS 1996. LNCS, vol. 1086, pp. 614-622. Springer, Heidelberg (1996)

10. Vu, K.-P.L., Minakata, K., Nguyen, J., Kraut, J., Raza, H., Battiste, V., Strybel, T.Z.: Situation Awareness and Performance of Student versus Experienced Air Traffic Controllers. In: Salvendy, G., Smith, M.J. (eds.) HCI International 2009. LNCS, vol. 5618, pp. 865-874. Springer, Heidelberg (2009)

11. Schneider, W.: Training High-Performance Skills: Fallacies and Guidelines. Hum. Factors 27(3), 285-300 (1985)

12. Sohn, M., Douglass, S.A., Chen, M., Anderson, J.R.: Characteristics of Fluent Skills in a Complex, Dynamic Problem-Solving Task. Hum. Factors 47, 742-752 (2005)

13. Carlson, R.A., Khoo, B.H., Elliot II, R.G.: Component Practice and Exposure to a Problem-Solving Context. Hum. Factors 32(3), 267-286 (1990)

14. Kurtz, S., Lee, T.D.: Part and Whole Perceptual-Motor Practice of a Polyrhythm. Neurosci. Lett. 338, 205-208 (2003) 
15. Lee, F.J., Anderson, J.R.: Does Learning a Complex task have to Be Complex?: A Study in Learning Decomposition. Cognitive Psychol 42, 267-316 (2001)

16. Young, M.D., Healy, A.F., Gonzalez, C., Dutt, V., Bourne Jr., L.E.: Effects of Training with Added Difficulties on RADAR Detection. Appl. Cognit. Psychol. (2010)

17. Dao, A.-Q.V., Brandt, S.L., Battiste, V., Vu, K.-P.L., Strybel, T., Johnson, W.W.: The Impact of Automation Assisted Aircraft Separation on Situation Awareness. In: Salvendy, G., Smith, M.J. (eds.) HCI International 2009. LNCS, vol. 5618, pp. 738-747. Springer, Heidelberg (2009)

18. Kraut, J.M., Kiken, A., Billinghurst, S., Morgan, C.A., Strybel, T.Z., Chiappe, D., Vu, K.P.L.: Effects of Data Communications Failure on Air Traffic Controller Sector Management Effectiveness, Situation Awareness, and Workload. To be presented at Human Computer Interaction International (2010)

19. Prevot, T.: Exploring the Many Perspectives of Distributed Air Traffic management: The Multi Aircraft Control System MACS. In: Proceedings of the HCI-Aero, pp. 149-254 (2002)

20. Canton, R., Refai, M., Johnson, W.W., Battiste, V.: Development and Integration of Human-Centered Conflict Detection and Resolution Tools for Airborne Autonomous Operations. In: Proceedings of the $15^{\text {th }}$ International Symposium of Aviation Psychology, Oklahoma State University, OK (2005) 